\title{
Effects of Ischemic Preconditioning of Different Intraoperative Ischemic Times of Vascularized Bone Graft Rabbit Models
}

\author{
Ahmad Sukari Halim ${ }^{1}$, Wan Syazli Rodzaian Wan Ahmad Kamal ${ }^{1,2}$, Norizal Mohd Noor ${ }^{2}$, \\ Shafie Abdullah ${ }^{1}$ \\ ${ }^{1}$ Reconstructive Sciences Unit, School of Medical Sciences, Universiti Sains Malaysia, Kubang Kerian, Kelantan; ${ }^{2}$ Faculty of Medicine, MARA \\ Institute of Technology University (UiTM), Sungai Buloh, Selangor, Malaysia
}

Background Ischemic preconditioning has been shown to improve the outcomes of hypoxic tolerance of the heart, brain, lung, liver, jejunum, skin, and muscle tissues. However, to date, no report of ischemic preconditioning on vascularized bone grafts has been published.

Methods Sixteen rabbits were divided into four groups with ischemic times of 2, 6, 14, and 18 hours. Half of the rabbits in each group underwent ischemic preconditioning. The osteomyocutaneous flaps consisted of the tibia bone, from which the overlying muscle and skin were raised. The technique of ischemic preconditioning involved applying a vascular clamp to the pedicle for 3 cycles of 10 minutes each. The rabbits then underwent serial plain radiography and computed tomography imaging on the first, second, fourth, and sixth postoperative weeks. Following this, all of the rabbits were sacrificed and histological examinations were performed.

Results The results showed that for clinical analysis of the skin flaps and bone grafts, the preconditioned groups showed better survivability. In the plain radiographs, except for two non-preconditioned rabbits with intraoperative ischemic times of 6 hours, all began to show early callus formation at the fourth week. The computed tomography findings showed more callus formation in the preconditioned groups for all of the ischemic times except for the 18hour group. The histological findings correlated with the radiological findings. There was no statistical significance in the difference between the two groups.

Conclusions In conclusion, ischemic preconditioning improved the survivability of skin flaps and increased callus formation during the healing process of vascularized bone grafts.

Keywords Free tissue flaps / Ischemic preconditioning / Microsurgery
Correspondence:

Ahmad Sukari Halim

Reconstructive Sciences Unit, School of Medical Sciences, Universiti Sains Malaysia, Kubang Kerian, Kelantan,

Malaysia

Tel: $+609-7676000$

Fax: +609-7656434

E-mail: ashalim@kb.usm.my

This study was supported by Universiti Sains Malaysia short-term grant (No USM/PPSP $\circledast / 2010 / J K P-43[43.3(B)]$ ).

This paper was presented during the Annual Scientific Meeting of the Royal Australasian College of Surgeons, Kuala Lumpur held in May 6-10, of which the abstract has been published in the supplementary issue of the (ANZ) Journal of Surgery, Volume 82 (Supplement 1) page 138.

No potential conflict of interest relevant to this article was reported.

Received: 3 Jun 2013 • Revised: 6 Aug 2013 • Accepted: 7 Aug 2013

pISSN: 2234-6163 • elSSN: 2234-6171 • http://dx.doi.org/10.5999/aps.2013.40.6.687 • Arch Plast Surg 2013;40:687-696

\section{INTRODUCTION}

The management of complex defects involving the loss of a huge segment of bone has long been very challenging to reconstruc- tive surgeons. Such defects can either be due to a motor vehicle accident, tumor resection, or a congenital etiology [1]. Among the options of reconstruction available nowadays are vascularized as well as non-vascularized bone grafts.

Copyright $(\odot 2013$ The Korean Society of Plastic and Reconstructive Surgeons

This is an Open Access article distributed under the terms of the Creative Commons Attribution Non-Commercial License (http://creativecommons.org/

licenses/by-nc/3.0/) which permits unrestricted non-commercial use, distribution, and reproduction in any medium, provided the original work is properly cited.

www.e-aps.org 
Ischemic preconditioning during the transfer of a vascularized bone graft is a novel technique studied in this paper to enhance the viability of the bone cells after prolonged intraoperative ischemic times. Ischemic preconditioning has been studied extensively and found to be an effective method of inducing hypoxic tolerance in heart, brain, lung, liver, jejunum, skin, and muscle tissues [2-7]. However, no study has been done so far to determine the applicability of this technique during the transfer of a vascularized bone graft. Thus, our aim was to study the effect of ischemic preconditioning during the transfer of a vascularized bone graft.

\section{METHODS}

Approval was obtained for this study from the Animal Ethics Committee of Malaysia Science University, and it was conducted according to the rules and requirements for an animal laboratory study as determined by the committee. Eighteen albino New Zealand male rabbits with an average weight of 4,000 g each were used. The first two rabbits were dissected to familiarize the researchers with their anatomy. The remaining 16 rabbits were divided into 4 groups comprising 4 rabbits each. Each of these groups was then subdivided into 2 subgroups comprising 2 rabbits in each group, with one subgroup undergoing ischemic preconditioning.

Each rabbit underwent surgery through a lateral incision approach to one hindlimb to locate the distal femur (Fig. 1). Following this, the saphenous artery, which is the vascular pedicle to the bone graft, was dissected up to the femoral vessel. Oste-

\section{Fig. 1. Preoperative marking of the flap design}

Preoperative marking of the flap design on the rabbit's right hindlimb. The size of the skin paddle was measured and the rabbit underwent surgery under general anesthesia with isoflurane at the animal house operating theater. The flap was based on the medial aspect of the hindlimb.

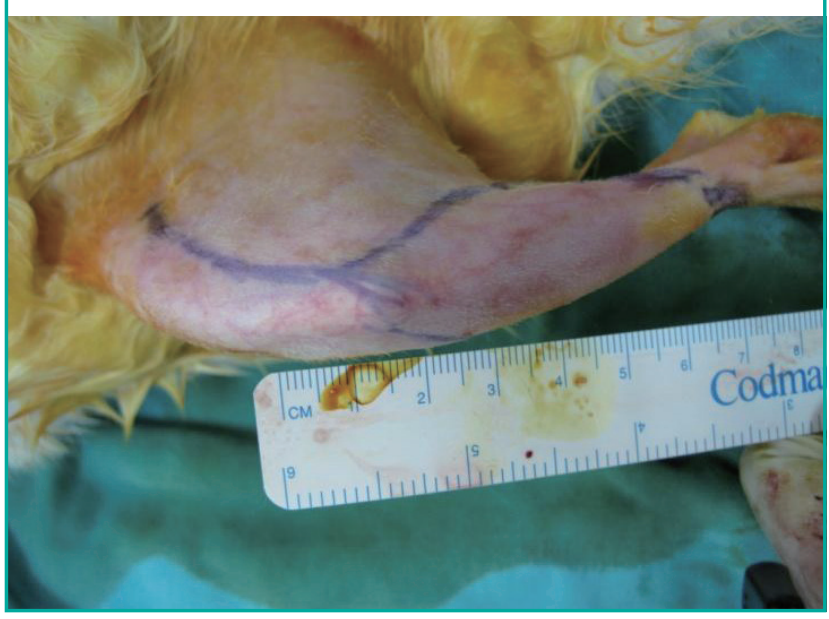

otomy was then performed on the distal portion of the femur based on this vascular pedicle and the vascularized bone graft was elevated together with an overlying skin paddle (Fig. 2). The vascular pedicle was clamped for the designated ischemic time before the blood flow was re-established and the bone graft replaced into the original site and fixed using K-wires (Fig. 3). Skin paddle size was standardized at $2 \mathrm{~cm}^{2}$ and the bone graft length at $2 \mathrm{~cm}$. Only $1 \mathrm{~K}$-wire was used for each limb. Finally, the skin paddle was resutured back to the surrounding skin. Out of the total of 18 rabbits, 2 rabbits were earlier dissected to become familiar with the anatomical findings. The remaining 16 rabbits were divided into 4 groups comprising four rabbits each. The rabbits in group 1 had an intraoperative ischemic time of 2 hours; group 2, 6 hours; group 3, 14 hours; and group 4,

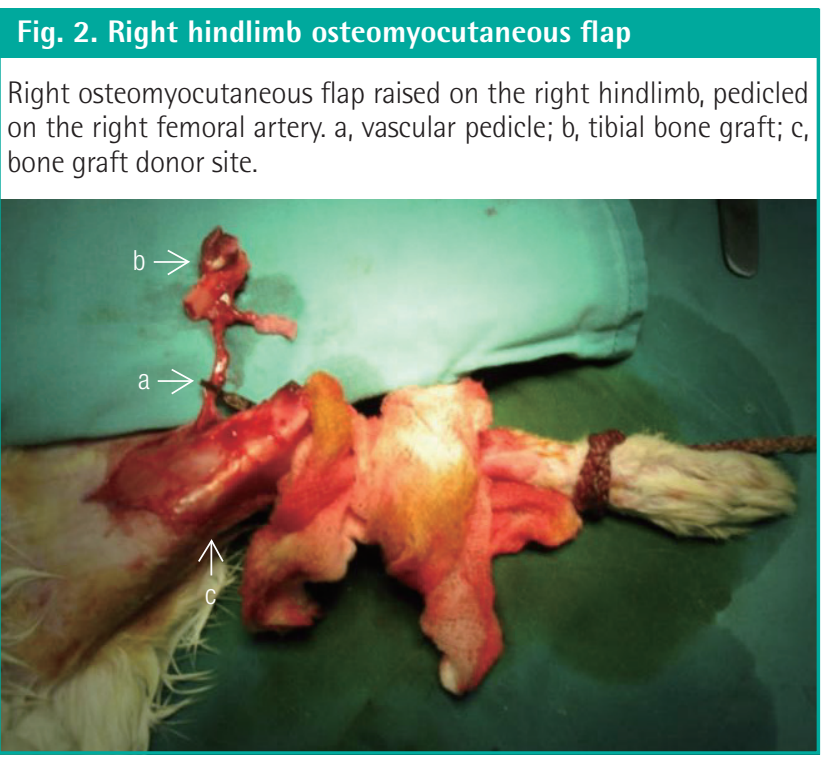

Fig. 3. Vascular clamp applied to the femoral vessel

Vascular clamp being applied to the femoral vessel to induce ischemia. This clamp was released then re-clamped to induce the state of ischemic preconditioning. Subsequently, the clamp was then applied continuously to simulate a free flap model. a, vascular clamp on the flap pedicle.

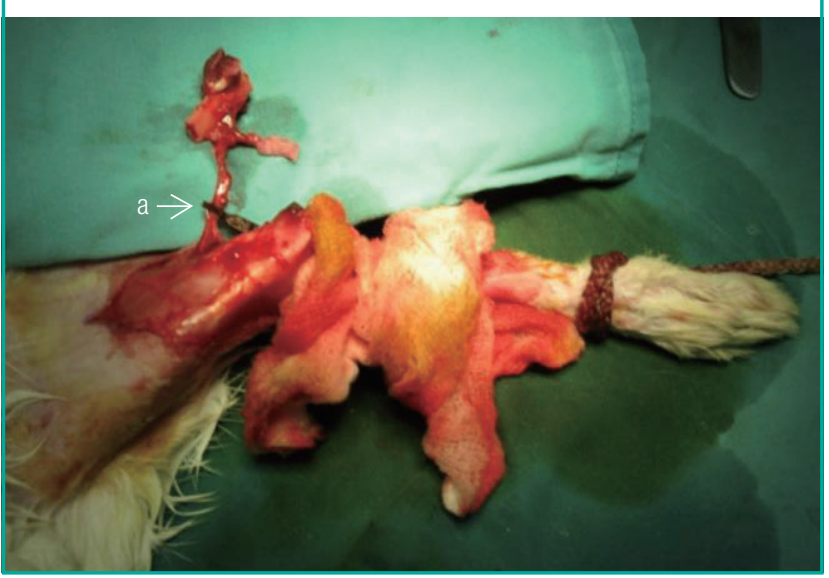


18 hours. Immediately following this, two of the rabbits of each subgroup underwent ischemic preconditioning. There was no delay in time prior to subjecting the rabbits to ischemic preconditioning. The technique of ischemic preconditioning involved applying a vascular clamp on the pedicle for 3 cycles of 10 minutes each, with a release of clamping of 10 minutes between each cycle.

The percentage of skin flap necrosis was compared among the experimental group by using planimetry. These assessments were performed on day 2 , day 4 , and day 6 following the procedure. All of the findings were documented in a standard form. Macroscopic assessment of bone grafts was later performed by two blinded assessors on the sixth week after they were harvested prior to sectioning for histological analysis. The assessment for the bone grafts included the gross appearance of the proximal and distal host-graft junctions in terms of bony union as well as the viability of the bone graft shafts themselves. Bony union was defined by an immobile host-graft junction with the presence of a bridging callus. The bone grafts were defined as viable based on the presence of healthy tissues and absence of necrotic segments.

Each animal underwent radiological evaluation, just after, 2 weeks after, 4 weeks after, and 6 weeks after the procedure when

\section{Fig. 4. Radiograph showing K-wire in place}

A plain radiograph taken to confirm the location of the K-wire that was inserted to fix the bone graft. Subsequent periodic radiographs were then performed for each operated limb to assess the radiological changes using a modified Sao Paolo score.

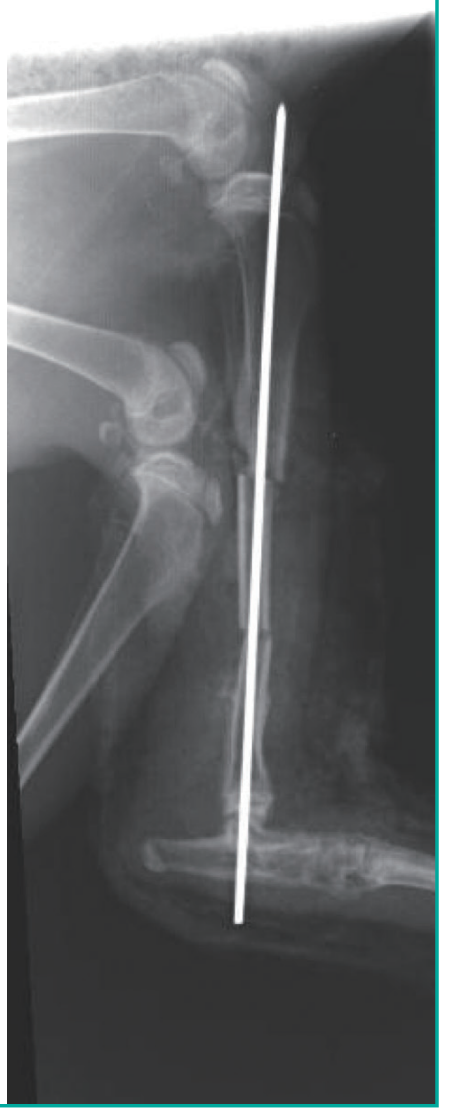

significant bone healing was anticipated (Fig. 4). A modification of the Sao Paolo classification system was used for the interpretation of the plain radiograph images. The computed tomography $(\mathrm{CT})$ scans were analyzed using the software OsiriX ver. 3.5.1 64-bit. The soft tissue window was chosen, as it showed better delineation of the callus formed. A free hand technique was chosen to outline the area of the callus for each slice, and then the mean reading of the callus area was calculated. Statistical calculation using the Mann-Whitney test was then used to determine the significance of differences between the study and control groups.

All of the rabbits were sacrificed at the end of the sixth week. Decalcification was achieved by immersing the entire tibia shafts in $5 \%$ nitric acid. Vertical sections from the proximal host- graft junctions were made. These were stained with hematoxylin and eosin. Assessment of each slide was performed under mi- croscope to determine the average number of viable osteocytes per 10 high power fields (HPF); whether the callus was mini- mal, moderate, or abundant; the appearance of the Haversian system; and whether the marrow appeared viable or non-viable (Figs. 5-8).

\section{RESULTS}

\section{Skin flaps}

The difference in skin flap changes between the preconditioned and non-preconditioned groups were more marked after a longer ischemic time (Table 1). In the 2 hour ischemic groups, all of the 4 rabbits had good survival of the skin flaps at day 2, day 4, and day 6 postoperatively. In most of the 6-hour ischemic time groups, the non-preconditioned skin flap had less than $25 \%$ viable area, whereas for the preconditioned groups, most of the skin flaps had areas of viability between $26 \%$ and $50 \%$. For ischemic times up to 14 hours, in the non-preconditioned groups, one rabbit showed $100 \%$ necrosis of the skin flap while another rabbit had less than a $25 \%$ viable area of the skin flap. On the other hand, no rabbit had $100 \%$ skin necrosis in the preconditioned groups. Similarly, for the 18-hour ischemic time groups, in the non-preconditioned rabbits, all skin flaps had 100\% necrosis. On the other hand, for the preconditioned rabbits in this group, both rabbits showed areas of viable skin on the flaps.

\section{Bone grafts}

The appearance of the bone grafts were examined in three sections, namely the proximal host-graft junction, the distal hostgraft junction, and the bone graft shaft itself (Table 2). In the shorter ischemic time groups of 2 hours and 6 hours, not much difference was noted in the gross appearance of the bone grafts between the preconditioned and non-preconditioned rabbits. 
Fig. 5 . Callus formation as seen on the bone grafts

Varying extents of callus formation of the bone in the postoperative period. (A) Minimal callus formation and (B) abundant callus formation. Microscopy $4 x$. These slides were stained with hematoxylin and eosin.

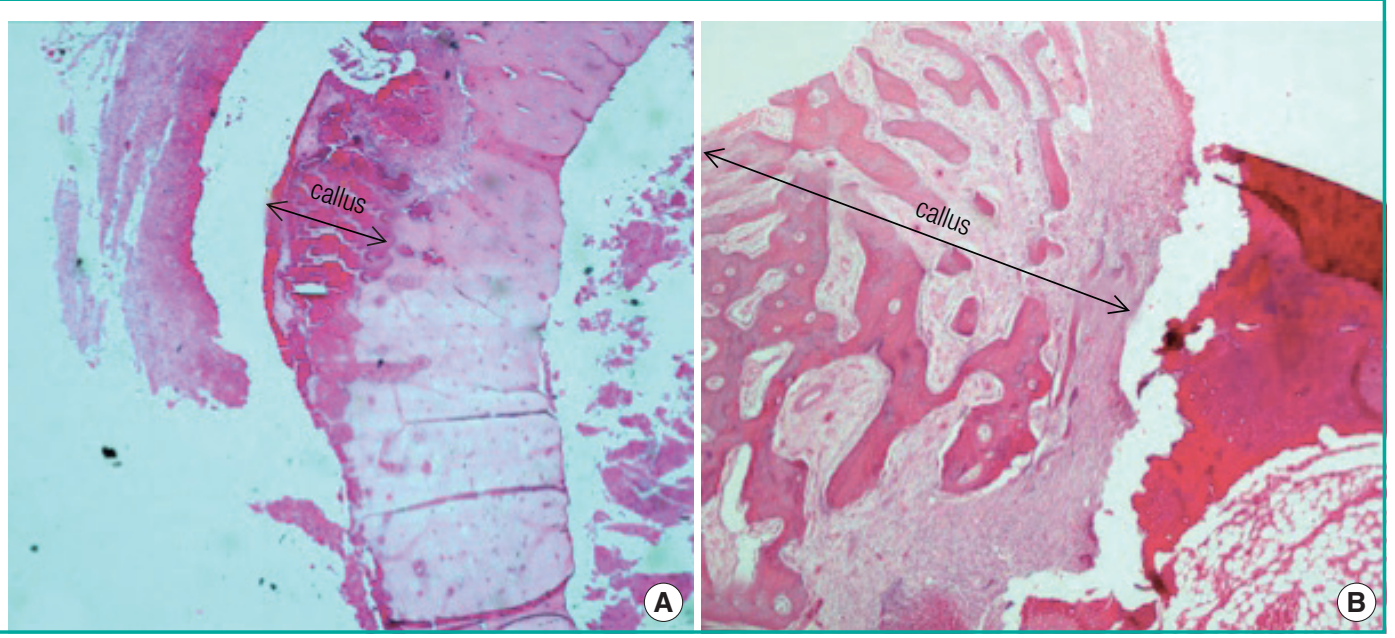

Fig. 6. Osteoblasts and osteocytes as seen on histological examination

Viable and non-viable osteoblasts $(\mathrm{OB})$ and osteocytes (OC) of the bone in the postoperative period. (A) Viable osteoblasts and osteocytes $(\times 20)$ and $(B)$ nonviable osteoblasts and osteocytes $(\times 40)$. These slides were stained with hematoxylin and eosin.
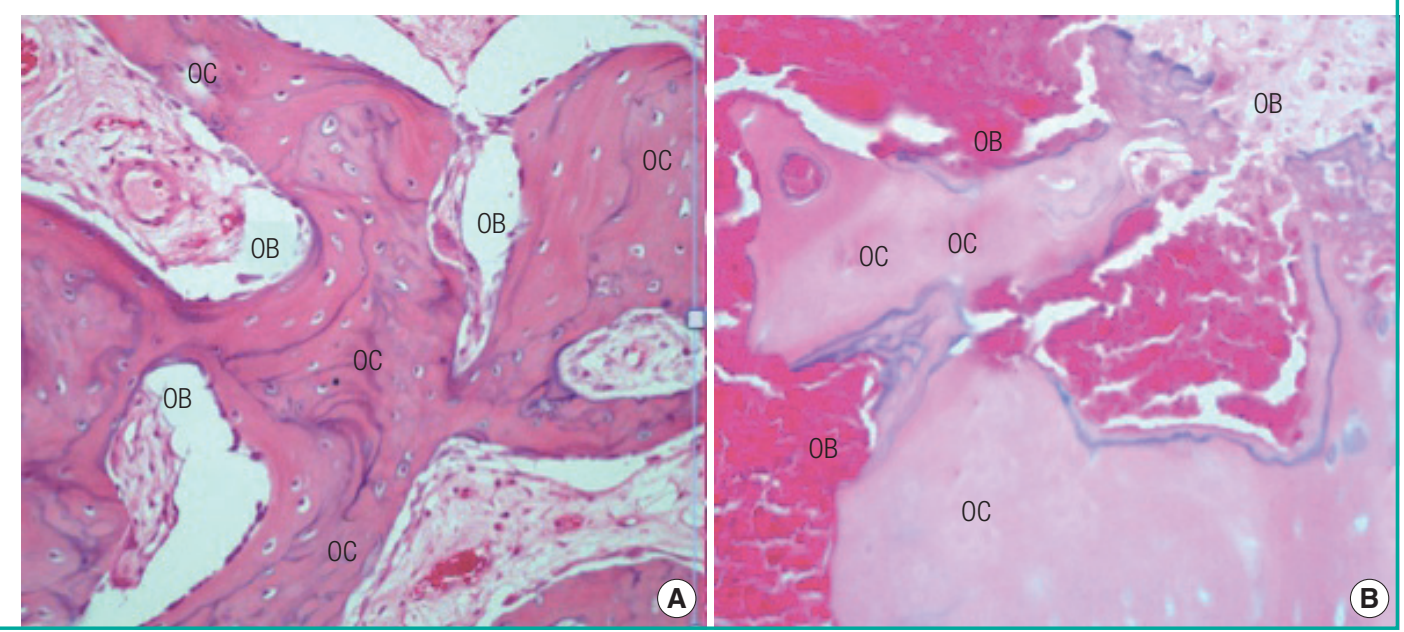

Fig. 7. Bone marrow findings as seen on histological examination

Viable and non-viable marrow content $(\mathrm{M})$ of the bone in the postoperative period. (A) Viable marrow content, with hematopoietic cells (4x) and (B) non-viable marrow content, filled mainly by adipose tissue (4x). These slides were stained with hematoxylin and eosin.
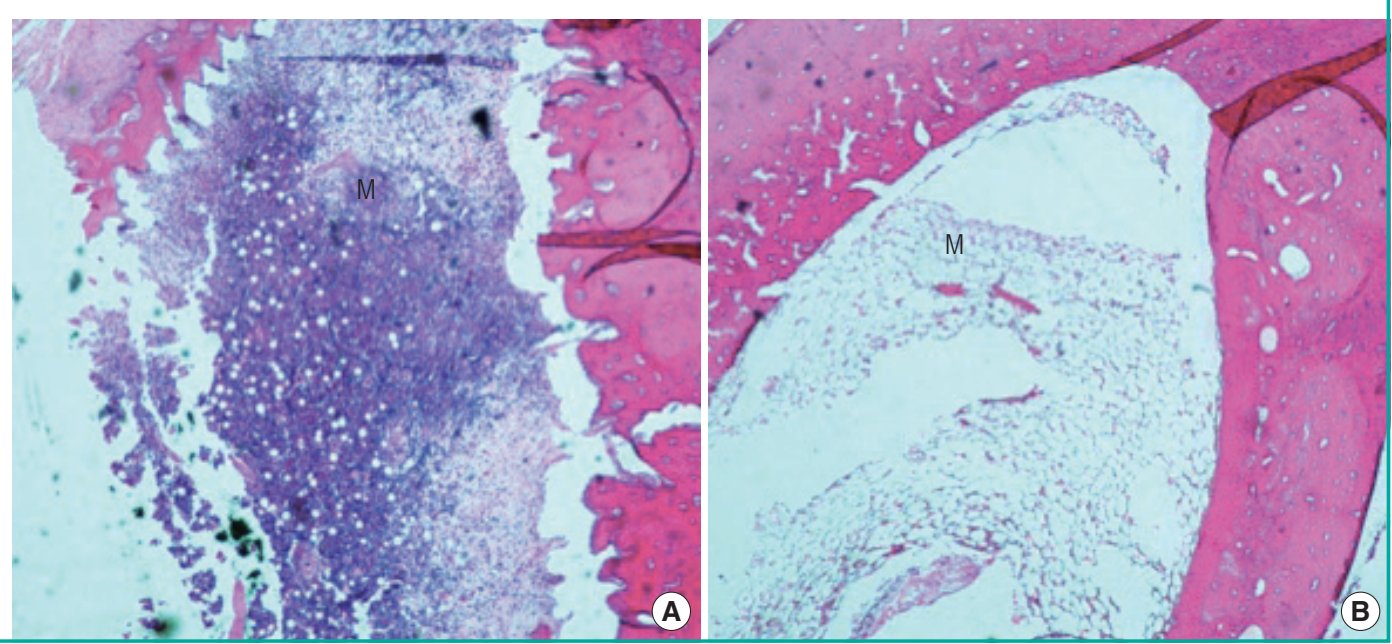


\section{Fig. 8. Computed tomography analysis of callus formation}

Freehand technique used for analysis of callus formation using computed tomography images. The callus area calculated for each slide can be seen.

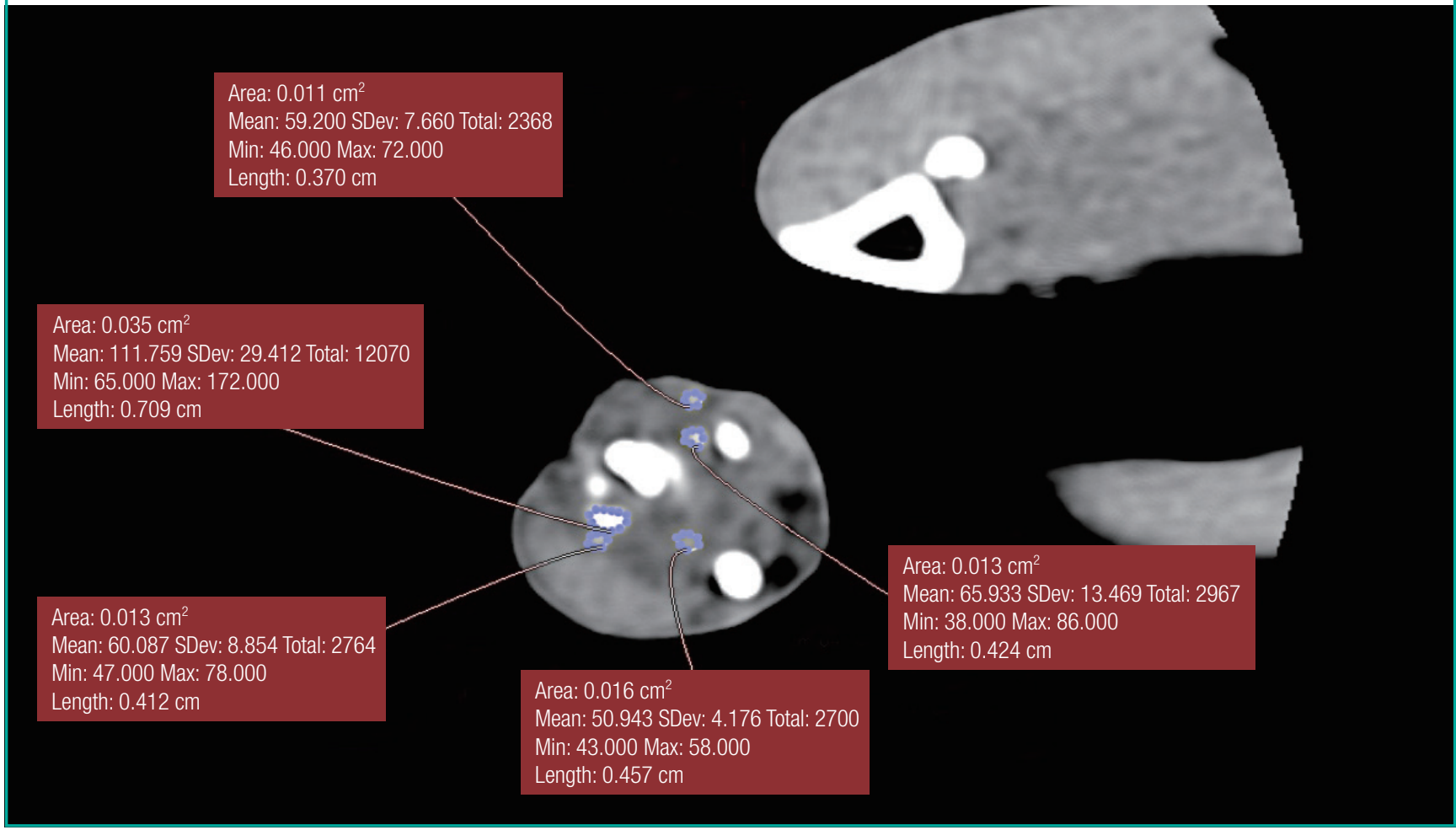

Table 1. Macroscopic appearance of skin flaps

\begin{tabular}{|c|c|c|c|c|c|c|}
\hline \multirow{2}{*}{ Preconditioning } & \multicolumn{2}{|c|}{ Day 1} & \multicolumn{2}{|c|}{ Day 5} & \multicolumn{2}{|c|}{ Day 7} \\
\hline & No & Yes & No & Yes & No & Yes \\
\hline $2 \mathrm{hr}$ & D & D & $D$ & D & C & $D$ \\
\hline $2 \mathrm{hr}$ & D & $\mathrm{D}$ & D & D & D & $\mathrm{D}$ \\
\hline $6 \mathrm{hr}$ & A & B & A & B & A & $B$ \\
\hline $6 \mathrm{hr}$ & B & B & A & B & A & $B$ \\
\hline $14 \mathrm{hr}$ & A & $A$ & A & A & A & A \\
\hline $14 \mathrm{hr}$ & $100 \%$ Non-viable & $A$ & $100 \%$ Non-viable & $A$ & $100 \%$ Non-viable & $A$ \\
\hline $18 \mathrm{hr}$ & $100 \%$ Non-viable & $A$ & $100 \%$ Non-viable & A & $100 \%$ Non-viable & $A$ \\
\hline $18 \mathrm{hr}$ & $100 \%$ Non-viable & $A$ & $100 \%$ Non-viable & A & $100 \%$ Non-viable & $A$ \\
\hline
\end{tabular}

Macroscopic examination of non-preconditioned skin flaps. Area of skin flap survival: A, less than $25 \%$; B, $26 \%$ to $50 \%$; C, $51 \%$ to $75 \%$; and D, $76 \%$ to $100 \%$. The difference in skin flap viability became more obvious in the groups with 14-hour and 18-hour ischemic times.

For non-preconditioned bone grafts in the longer ischemic times of the 14- and 18-hour groups, three of the rabbits showed union only at the proximal host-graft junctions whereas one rabbit in the 14-hour group showed non-union at both the proximal and distal junctions. On the other hand, in the ischemic preconditioned rabbits, all of the bone grafts appeared viable with union in both the proximal and distal host-graft junctions. The difference in the bone grafts' appearance was less obvious in the 18-hour groups.

\section{Radiological examination}

In the plain radiograph review, no callus was seen in the first imaging taken in the week for any of the rabbits (Table 3). Except for two rabbits with intraoperative ischemic times of 6 hours, the rest of the radiographs began to show early callus formation in the radiographs of 4 weeks. In the radiographs of 6 weeks, most of the bone grafts showed dense callus with signs of fracture healing. The mean of the total callus formation in each CT scan slice was calculated for each ischemic time group. The results showed more callus formation in the ischemic preconditioned animals of the 2-hour, 6-hour and 14-hour groups (Table 
4). However, for the 18-hour group, more callus tissue was seen in the non-preconditioned animals.

\section{Histological examination}

The slides of each rabbit were examined to examine the number of viable osteocytes, the characteristics of the callus, the appearance of the Haversian canal, and the viability of the marrow (Table 5). The left lower limbs for all of the rabbits were prepared as controls. At 6 weeks postoperatively, the number of surviving osteocytes for the non-preconditioned rabbits of the 2-hour, 6hour, and 14-hour ischemic groups were within 55 to 75 osteocytes per $10 \mathrm{HPF}$, except for the 18-hours ischemic time groups, which had fewer osteocytes. Similarly, for the preconditioned groups, the number of osteocytes for the different subgroups were within 55 to 75 osteocytes, including the 18-hour groups. The findings of moderate to abundant callus tissue were seen on the slides of all of the rabbits except for the non-preconditioned 18-hour rabbit, which had minimal callus tissue on both slides.
Table 3. Plain radiograph findings

\begin{tabular}{|lcccc|}
\hline Rabbits & First week & Second week & Third week & Fourth week \\
\hline 2 hr non-IP & 0 & 0 & 1 & 1 \\
2 hr non-IP & 0 & 2 & 3 & 4 \\
6 hr non-IP & 0 & 1 & 2 & 2 \\
6 hr non-IP & 0 & 2 & 3 & 4 \\
14 hr non-IP & 0 & 1 & 1 & 2 \\
14 hr non-IP & 0 & 1 & 1 & 2 \\
18 hr non-IP & 0 & 2 & 2 & 3 \\
18 hr non-IP & 0 & 1 & 2 & 2 \\
2 hr IP & 0 & 1 & 2 & 3 \\
2 hr IP & 0 & 1 & 2 & 3 \\
6 hr IP & 0 & 1 & 3 & 4 \\
6 hr IP & 0 & 1 & 2 & 3 \\
14 hr IP & 0 & 1 & 2 & 3 \\
14 hr IP & 0 & 1 & 2 & 3 \\
18 hr IP & 0 & 1 & 1 & 2 \\
18 hs IP & 0 & 1 & 1 & 2 \\
\hline Plain radiograps findings using a modified Sao Paolo scoring system showing \\
almost identical findings in between the preconditioned and non-preconditioned \\
groups. IP, ischemic preconditioning.
\end{tabular}

Table 2. Macroscopic appearance of bone grafts

\begin{tabular}{|c|c|c|c|c|c|c|}
\hline \multirow{2}{*}{ Preconditioning } & \multicolumn{2}{|c|}{ Proximal bone graft junction } & \multicolumn{2}{|c|}{ Distal bone graft junction } & \multicolumn{2}{|c|}{ Bone shaft } \\
\hline & No & Yes & No & Yes & No & Yes \\
\hline $2 \mathrm{hr}$ & Union & Union & Union & Union & Viable & Viable \\
\hline $2 \mathrm{hr}$ & Union & Union & Union & Union & Viable & Viable \\
\hline $6 \mathrm{hr}$ & Union & Union & Union & Union & Viable & Viable \\
\hline $6 \mathrm{hr}$ & Union & Union & Union & Union & Viable & Viable \\
\hline $14 \mathrm{hr}$ & Non-union & Union & Non-union & Union & Viable & Viable \\
\hline $14 \mathrm{hr}$ & Union & Union & Non-union & Union & Viable & Viable \\
\hline $18 \mathrm{hr}$ & Union & Union & Non-union & Non-union & Non-viable & Viable \\
\hline $18 \mathrm{hr}$ & Union & Union & Non-union & Non-union & Non-viable & Viable \\
\hline
\end{tabular}

Gross macroscopic examination of bone grafts at 6 weeks showing a difference in the proximal, distal, and marrow appearances, between the preconditioned and nonpreconditioned groups, mainly in the 18-hour group.

Table 4. Mean callus quantity seen on CT scan images and the statistical results for each group

\begin{tabular}{|c|c|c|c|c|c|}
\hline \multirow{2}{*}{ Comparison group } & \multicolumn{2}{|c|}{ Preconditioned } & \multicolumn{2}{|c|}{ Non-preconditioned } & \multirow{2}{*}{ P-value } \\
\hline & Median & Range & Median & Range & \\
\hline 2 hr/wk 2 & 0.174 & $0.041-0.256$ & 0.124 & $0.013-0.591$ & 0.439 \\
\hline 2 hr/wk 4 & 0.198 & $0.054-0.835$ & 0.191 & $0.013-0.639$ & 1.000 \\
\hline 2 hr/wk 6 & 0.124 & $0.046-1.266$ & 0.175 & $0.015-0.443$ & 0.121 \\
\hline 6 hr/wk 2 & 0.180 & $0.018-0.310$ & 0.191 & $0.015-0.322$ & 1.000 \\
\hline $6 \mathrm{hr} / \mathrm{wk} 4$ & 0.208 & $0.019-0.561$ & 0.192 & $0.018-0.286$ & 0.439 \\
\hline 6 hr/wk 6 & 0.231 & $0.043-0.752$ & 0.203 & $0.033-0.326$ & 0.439 \\
\hline 14 hr/wk 2 & 0.185 & $0.035-0.470$ & 0.142 & $0.048-0.433$ & 0.439 \\
\hline 14 hr/wk 4 & 0.231 & $0.036-0.581$ & 0.203 & $0.070-0.428$ & 0.121 \\
\hline 14 hr/wk 6 & 0.220 & $0.063-0.693$ & 0.181 & $0.064-0.645$ & 1.000 \\
\hline 18 hr/wk 2 & 0.151 & $0.041-0.427$ & 0.153 & $0.033-0.462$ & 1.000 \\
\hline $18 \mathrm{hr} / \mathrm{wk} 4$ & 0.162 & $0.041-0.462$ & 0.180 & $0.025-0.548$ & 0.439 \\
\hline 18 hr/wk 6 & 0.192 & $0.027-0.602$ & 0.243 & $0.026-0.628$ & 0.439 \\
\hline
\end{tabular}


Table 5. Histological examination findings

\begin{tabular}{|lccc|}
\hline Rabbits & $\begin{array}{c}\text { No of viable } \\
\text { osteocytes per HPF }\end{array}$ & $\begin{array}{c}\text { Characteristics of } \\
\text { callus }\end{array}$ & Haversian canal \\
\hline 2 hr non-IP & 58 & 2 & mVOC \\
2 hr non-IP & 74 & 3 & mVOB \\
2 hr IP & 53 & 2 & mVOC \\
2 hr IP & 80 & 3 & mVOC \\
6 hr non-IP & 64 & 2 & mVOC \\
6 hr non-IP & 66 & 2 & mVOB \\
6 hr IP & 52 & 3 & mVOC \\
6 hr IP & 54 & 2 & mVOC \\
14 hr non-IP & 64 & 2 & MVOB \\
14 hr non-IP & 56 & 1 & mVOC \\
14 hr IP & 54 & 2 & mVOC \\
14 hr IP & 56 & 3 & AVOC \\
18 hr non-IP & 30 & 1 & mVOC \\
18 hr non-IP & 3 & 1 & mNVOC \\
18 hr IP & 52 & 2 & mVOC \\
18 hr IP & 50 & 2 & MVOC \\
\hline Histological examination of the findings comparing the preconditioned and non- \\
preconditioned groups at the 6th postoperative week showed variable results \\
between the two groups. HPF, high power field; IP, ischemic preconditioning; OC, \\
osteocytes; OB, osteoblasts; m, minimal; M, moderate; A, abundant; V, viable; NV, \\
non-viable. Grading for callus characteristics: 1, minimal; 2, moderate; and 3, \\
abundant.
\end{tabular}

The appearance of the Haversian systems showed the presence of viable osteocytes and osteoblasts for all of the slides except for one rabbit with non-preconditioned 18-hours ischemic time showing non-viable osteocytes.

\section{DISCUSSION}

The clinical usage of a free vascularized bone graft during the reconstruction of complex skeletal defects often requires a prolonged intraoperative ischemic time. This time is required during the harvesting of the bone graft and preparation of the recipient site, as well as the duration of the microvascular anastomosis [8]. Even though osteocytes are known to have a high level of tolerance to ischemic insults, these cells, as with any living cells, are still susceptible to the grievous results of prolonged intraoperative deprivation of the blood supply [9]. One of the methods that has been studied extensively to enhance the viability of flap tissues is ischemic preconditioning. Ischemic preconditioning effectively belongs on a spectrum with ischemic reperfusion injury on the other extreme end.

One well-documented event during ischemic reperfusion injury is the attachment of platelets and leukocytes to vascular endothelial cells. Leukocytes also migrate into the extravascular space, where they release oxygen radicals, peroxynitrite, and enzymes that can damage or degrade tissues, including the heat shock proteins. Intravascular deposition of fibrin can also occur during a ischemic reperfusion injury [10]. Other than this, there will be edema, causing an increase in the passage of macromolecules and fluid from the vascular to the extravascular compartment [9].

Experimentally, the heat shock response has been demonstrated to have a positive effect on flap survival by protecting tissue from ischemia or ischemia-reperfusion injury [11]. These proteins are a superfamily of highly conserved intracellular proteins that protect cells and whole organisms from various stresses. The resulting induction of the heat shock proteins provides the cells with cross-protection from subsequent injuries. Apart from the effects of heat shock proteins, ischemic preconditioning also acts via the action of adenosine triphosphate (ATP)- sensitive potassium channels, arteriolar dilatation, iron regulatory protein, and the release of substances after prolonged ischemia such as adenosine, bradykinin, opioids, and oxygen radicals $[2,12$ $15]$.

As this was a pilot study using an animal model, the sample size was small in number. Berggren et al. [8] have shown the effect of prolonged ischemic time on osteocytes and osteoblasts in vascularized bone grafts anastomosed through microsurgical techniques. The ischemic time varied from 90 minutes to 48 hours. His results showed that a vascularized bone graft could survive an ischemic time of up to 25 hours. We have chosen an intraoperative ischemic time of between 2 hours and 18 hours as this, in our opinion, closely resembles the operative times in clinical settings.

The subsequent pedicle clamping technique was used instead of microsurgical anastomosis for several reasons. Even though microsurgical anastomosis would more closely resemble the actual clinical settings of free tissue transfer, there were several confounding factors that would affect the outcome of the procedure. This technique would consume a great deal of operative time to complete in each rabbit, and even after successful completion of the anastomosis, the flap would be exposed to the risk of arterial or venous thrombosis or anastomotic breakdown further requiring flap reexploration [16]. Early detection of any arterial or venous problem would be very difficult and even if we managed to detect it, emergency flap re-exploration might not be possible to perform for logistical reasons. This will further complicate the procedure and delay the completion of this project. Thus, through clamping of the flap pedicle, we were also able to create a complete cessation of the blood supply to and from the flap, essentially producing a state of ischemia during flap transfer, without all the problems of microvascular anastomosis as mentioned earlier.

In comparing between the preconditioned and non-preconditioned animals, in the 2-hour groups, the areas of skin flap survival were equal for the two groups during all wound inspec- 
tions. The 2-hour ischemic time was relatively too short to influence the survival of the keratinocytes. In the 6-hour ischemic time groups, better survival was seen in the preconditioned groups. This duration of ischemia may mimic more actual clinical scenarios. This finding correlates with most of the other studies on the effects of ischemic preconditioning on skin flaps [15].

For the longer ischemic time groups of 14 and 18 hours, it was found that all of the skin flaps in the non-preconditioned rabbits were not viable. However, in the preconditioned group, both rabbits showed small areas of skin flap survival. Zahir et al. [17] examined the effects of ischemic preconditioning on 220 rats via 100 transverse rectus abdominis myocutaneous flaps and 120 dorsal cutaneous flaps. His results showed that for the skin flap model, statistical significance of the survival area difference was reached at 6,10, and 14 hours of ischemia.

In rabbits, good union after fracture healing was seen at the sixth postoperative week [17]. Plain radiograph analysis of the 2 groups was performed using a modified scoring system based on the Sao Paolo classification [18]. This scoring system was used because of its simplicity in interpreting the results. We have modified it by combining some categories that were difficult to clearly differentiate. Inter-observer variability during the assessment of plain radiographs has been reported to be between $20 \%$ and $25 \%$ [19].

The process of bone healing followed the natural process with mostly dense callus tissue seen in the sixth week radiographs. Early callus formation was beginning to be seen in all of the groups after the fourth postoperative week. The findings from the plain radiographs trace the process of fracture healing, with changes starting to be visible from the third to fourth week after fracture. This correlates with the physiological phase of wound healing, when the hard callus stage usually starts [20]. In this study, at the sixth week, for the 2, 6, and 14 ischemic hour groups, a higher score for dense callus and signs of fracture healing were seen in the preconditioned animals. The difference was greater in the groups with a shorter ischemic period than the 18-hour group. We postulated that in the 18-hour group, the extreme ischemic time resulted in widespread osteocyte death even before preconditioning was commenced. However, future studies to specifically prove this theory are warranted.

In this study, a compounding factor that needed to be taken into consideration was the presence of internal fixation in between the fracture segments. Internal fixation affects the mechanical and biological environment of a fracture, hence affecting the amount of callus form and cortical bridging. Primary healing occurs only when anatomic restoration of the fracture fragments takes place, by rigid internal fixation, and when the stability of fracture reduction is ensured by a substantial de- crease in interfragmentary strain. Under these conditions, boneresorbing cells on one side of the fracture show a tunnelling resorptive response, whereby they re-establish new Haversian systems by providing pathways for the penetration of blood vessels. The amount of callus formation is influenced by the relative stability of the fracture fragments. The more motion there is at a fracture site, the larger a callus is needed to prevent this motion [21].

The outcome showed an increasing quantity of callus formation from week 2 to week 6 in all of the groups. In comparison between with the animals that underwent ischemic preconditioning, as seen on the CT scans, those without preconditioning appeared to have more callus tissue formation in the subgroups of 2 hours, 6 hours, and 14 hours. However, the non-preconditioned group showed more callus formation in the 18-hour group. Therefore, we postulated that at 18 hours, the initial number of non-viable osteocytes upon completion of the procedure was more than in the other groups; thus, unlike the others, they were not influenced by ischemic preconditioning. However, further studies looking into the histochemical changes after the respective ischemic times will objectively prove this. As for quantitative evaluation, the CT analyses showed good intraobserver and interobserver reproducibililty [22]. Only 16 rabbits were used, as this was a pilot study. As the data were non-parametric, we chose the Mann-Whitney test for statistical evaluation. This would inflate the type I errors by running so many tests at the same time, even though it may not be necessary to correct type I errors for this exploratory analysis. The results showed no significant difference between the preconditioned and nonpreconditioned groups. With a larger sample size, we would expect the result to be significantly different with more favorable outcomes in the ischemic preconditioned groups. We would have the ability to obtain more precise data by limiting errors caused by confounding factors, which are a limiting factor with a smaller sample size. Additional animals in each preconditioning time subgroup would also increase the power for calculation to determine the significance of the statistical results.

The technique of ischemic preconditioning used in this study involved the application of vascular clamps on the flap pedicles for three cycles of 10 minutes each. We based our technique on a study performed by Zahir et al. [17] that applied the same preconditioning protocol during the transfer of a pedicled rectus abdominis myocutaneous flap in a rat model. The ischemic preconditioning protocol used for this study allowed an even distribution of time for ischemic preconditioning and reperfusion of the flap models. The period of 10 minutes was also deemed adequate to expose the osteocytes to the ischemic insult without creating the unnecessary damage to the cells that an excessive 
exposure would. This is based on an ischemic preconditioning model of skin flaps by Zahir et al., [17] as no other studies of ischemic preconditioning on bone grafts have been reported. We did not use a shorter ischemic time, as it might be too brief to create the preconditioning effects to the bone cells.

A myocyte model of ischemic preconditioning has been shown to lead to rapid phosphorylation of potassium-adenosine triphosphate channels, which leads to the cardioprotective effects of $\mathrm{K}^{+}$efflux. Ischemic preconditioning has also been shown to result in heat shock-mediated arteriolar dilation associated with an increase in volumetric blood flow in individual capillaries. The increased capillary volume flow is caused by capillary dilation rather than increased red blood cell velocity. Katori et al. [23] demonstrated that ischemic preconditioning also enhanced the action of heat shock proten HSP-32. Competitive inhibition of HSP-32 function completely abrogated the protection from necrosis. In addition to this, previous studies on renal and myocardial reperfusion and re-oxygenation have indicated that HSP-32 attenuates apoptotic cell death. Hutter et al. [24] demonstrated that in rabbits, the brief period of preconditioning ischemia and reperfusion releases adenosine, bradykinin, opioids, and oxygen radicals. The combined effect of the release of these substances on $\mathrm{G}$ proteins and the cell's phospholipases induces the translocation and activation of the $\varepsilon$ isozyme of protein kinase $\mathrm{C}$. Protein kinase $\mathrm{C}$ appears to be the first element of a complex kinase cascade that is activated during the prolonged ischemia in preconditioned hearts. Current evidence indicates that this cascade contains at least one tyrosine kinase and ultimately leads to the activation of $\mathrm{p} 38$ mitogen-activated protein kinase. This substance is one of the proteins that controls actin filament polymerization, and, therefore, affects the integrity of the cytoskeleton [10].

\section{REFERENCES}

1. Shadgan B, Menon M, O’Brien PJ, et al. Diagnostic techniques in acute compartment syndrome of the leg.J Orthop Trauma 2008;22:581-7.

2. Berenshtein E, Vaisman B, Goldberg-Langerman C, et al. Roles of ferritin and iron in ischemic preconditioning of the heart. Mol Cell Biochem 2002;234-235:283-92.

3. Barone FC, White RF, Spera PA, et al. Ischemic preconditioning and brain tolerance: temporal histological and functional outcomes, protein synthesis requirement, and interleukin-1 receptor antagonist and early gene expression. Stroke 1998;29:1937-50.

4. Soncul H, Oz E, Kalaycioglu S. Role of ischemic preconditioning on ischemia-reperfusion injury of the lung. Chest
1999;115:1672-7.

5. Peralta C, Bartrons R, Serafin A, et al. Adenosine monophosphate-activated protein kinase mediates the protective effects of ischemic preconditioning on hepatic ischemiareperfusion injury in the rat. Hepatology 2001;34:1164-73.

6. Davis JM, Gute DC, Jones S, et al. Ischemic preconditioning prevents postischemic $\mathrm{P}$-selectin expression in the rat small intestine. Am J Physiol 1999;277:H2476-81.

7. Gurke L, Mattei A, Chaloupka K, et al. Mechanisms of ischemic preconditioning in skeletal muscle. J Surg Res 2000; 94:18-27.

8. Berggren A, Weiland AJ, Ostrup LT, et al. Microvascular free bone transfer with revascularization of the medullary and periosteal circulation or the periosteal circulation alone. A comparative experimental study.J Bone Joint Surg Am 1982; 64:73-87.

9. Kalebo P, Johansson C, Albrektsson T. Temporary bone tissue ischemia in the hind limb of the rabbit. A vital microscopic study. Arch Orthop Trauma Surg 1986;105:321-5.

10. Menger MD, Laschke MW, Amon M, et al. Experimental models to study microcirculatory dysfunction in muscle ischemia-reperfusion and osteomyocutaneous flap transfer. Langenbecks Arch Surg 2003;388:281-90.

11. Opie LH. Myocardial ischaemia: metabolism and its modification. S Afr Med J 1987;72:740-7.

12. Marijic J, Stowe DF, Turner LA, et al. Differential protective effects of halothane and isoflurane against hypoxic and reoxygenation injury in the isolated guinea pig heart. Anesthesiology 1990;73:976-83.

13. Rucker M, Schafer T, Roesken F, et al. Local heat-shock priming-induced improvement in microvascular perfusion in osteomyocutaneous flaps is mediated by heat-shock protein 32. Br J Surg 2001;88:450-7.

14. Schott RJ, Rohmann S, Braun ER, et al. Ischemic preconditioning reduces infarct size in swine myocardium. Circ Res 1990;66:1133-42.

15. Manson PN, Anthenelli RM, Im MJ, et al. The role of oxygen-free radicals in ischemic tissue injury in island skin flaps. Ann Surg 1983;198:87-90.

16. Yoshimura M, Shimamura K, Iwai Y, et al. Free vascularized fibular transplant. A new method for monitoring circulation of the grafted fibula. J Bone Joint Surg Am 1983;65:1295301.

17. Zahir KS, Syed SA, Zink JR, et al. Ischemic preconditioning improves the survival of skin and myocutaneous flaps in a rat model. Plast Reconstr Surg 1998;102:140-50.

18. Sharifi P, Yunessi F, Sasani M, et al. The effects of transcutaneous electrical stimulation on the healing of radial fracture 
in rabbit. Am J Anim Vet Sci 2006; 1:13-6.

19. Turek SL. Reparo da fratura simples de um osso longo. In: Turek SL, editor. Ortopedia: princípios e sua aplicacao. Sao Paulo: Manole; 1991. p.58-62.

20. Whelan DB, Bhandari M, McKee MD, et al. Interobserver and intraobserver variation in the assessment of the healing of tibial fractures after intramedullary fixation. J Bone Joint Surg Br 2002;84:15-8.

21. Greenbaum MA, Kanat IO. Current concepts in bone healing. Review of the literature. J Am Podiatr Med Assoc 1993;83:123-9.
22. Kawai T, Murakami S, Hiranuma H, et al. Radiographic changes during bone healing after mandibular fractures. $\mathrm{Br} \mathrm{J}$ Oral Maxillofac Surg 1997;35:312-8.

23. Katori M, Buelow R, Ke B, et al. Heme oxygenase-1 overexpression protects rat hearts from cold ischemia/reperfusion injury via an antiapoptotic pathway. Transplantation 2002; 73:287-92.

24. Hutter MM, Sievers RE, Barbosa V, et al. Heat-shock protein induction in rat hearts. A direct correlation between the amount of heat-shock protein induced and the degree of myocardial protection. Circulation 1994;89:355-60. 